\title{
Bismuthene on a SiC Substrate: A Candidate for a New High-Temperature Quantum Spin Hall Paradigm
}

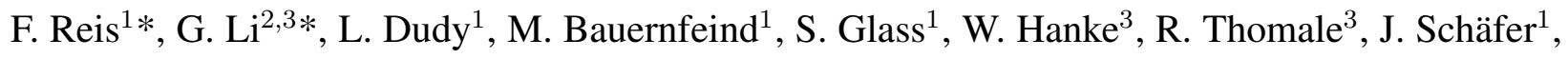 \\ and R. Claessen ${ }^{1}$ \\ ${ }^{1}$ Physikalisches Institut and Röntgen Center for Complex Material Systems, Universität Würzburg, \\ D-97074 Würzburg, Germany \\ ${ }^{2}$ Institute of Solid State Physics, Vienna University of Technology, A-1040 Vienna, Austria \\ ${ }^{3}$ Institut für Theoretische Physik und Astrophysik, Universität Würzburg, D-97074 Würzburg, \\ Germany \\ * These authors contributed equally to this work.
}

Quantum spin Hall (QSH) materials promise revolutionary device applications based on dissipationless propagation of spin currents. They are two-dimensional (2D) representatives of the family of topological insulators, which exhibit conduction channels at their edges inherently protected against scattering. Initially predicted for graphene ${ }^{1 / 2}$, and eventually realized in HgTe quantum wells ${ }^{34}$, in the QSH systems realized so far $\frac{5,6}{6}$, the decisive bottleneck preventing applications is the small bulk energy gap of less than $30 \mathrm{meV}$, requiring cryogenic operation temperatures in order to suppress detrimental bulk contributions to the edge conductance ${ }^{788}$. Room-temperature functionalities, however, require much larger gaps. Here we show how this can be achieved by making use of a new QSH paradigm based on substratesupported atomic monolayers of a high- $Z$ element. Experimentally, the material is synthe- 
sized as honeycomb lattice of bismuth atoms, forming "bismuthene", on top of the wide-gap substrate $\mathrm{SiC}(0001)$. Consistent with the theoretical expectations, the spectroscopic signatures in experiment display a huge gap of $\approx 0.8 \mathrm{eV}$ in bismuthene, as well as conductive edge states. The analysis of the layer-substrate orbitals arrives at a QSH phase, whose topological gap - as a hallmark mechanism - is driven directly by the atomic spin-orbit coupling (SOC). Our results demonstrate how strained artificial lattices of heavy atoms, in contact with an insulating substrate, can be utilized to evoke a novel topological wide-gap scenario, where the chemical potential is located well within the global system gap, ensuring pure edge state conductance. We anticipate future experiments on topological signatures, such as transport measurements that probe the QSH effect via quantized universal conductance, notably at room temperature.

While the QSH phase as such is characterized by universal topological properties ${ }^{2}$, other aspects such as the bulk energy gap are material-specific, and depend on the combination of SOC (in elements of atomic number $Z$ scaling as $Z^{4}$ ), orbital hybridization, and the symmetries of the layer-substrate system. In QSH honeycomb layers mimicking graphene $\mathrm{e}^{1}$ (where minute SOC precludes a noticeable gap), one obvious strategy to generate enhanced gaps is to replace $\mathrm{C}$ by heavier atoms. For systems with the group-IV elements $\mathrm{Si}, \mathrm{Ge}$, and $\mathrm{Sn}$, predicted gaps are 2 meV $\mathrm{V}^{9}$, $24 \mathrm{meV}^{9}$, and $100 \mathrm{meV}^{10}$, respectively. This applies to hypothetical freestanding material - with poor chemical stability. Real-world variants require a supporting substrate for epitaxial synthesis, which also brings concurrent bonding interactions into play. Attempts were made to grow, e.g., silicene on a metallic Ag substrate ${ }^{1112}$, which however short-circuits any edge states of interest. 
An insulating $\mathrm{MoS}_{2}$ substrate can stabilize germanene $e^{13}$, yet the compressive strain renders the material a metal. Stanene flakes on $\mathrm{Bi}_{2} \mathrm{Te}_{3}$ are plagued by the same problem 14 .

Turning to group $\mathrm{V}$ of the Periodic Table, bismuth $(Z=83)$ must be expected to yield an even larger gap. It is predicted as topological non-trivial for thin free-standing layers $15 \mid-17$. The conceptual existence of a monolayer bismuthene QSH phase on a silicon substrate has been proposed $\frac{18}{18}$, and extended to $\mathrm{SiC}$ by modelling of the edge states 19 . However, experimental growth of a hexagonal single-layer Bi phase on $\mathrm{Si}(111)$ has failed $20 \mid 21$.

Our experimental realization of supported bismuthene employs a $\mathrm{SiC}(0001)$ substrate, on which we generate a $(\sqrt{3} \times \sqrt{3}) \mathrm{R} 30^{\circ}$ superstructure of $\mathrm{Bi}$ atoms in honeycomb geometry, as illustrated in Fig. 1a (details in Extended Data Fig. E1). The resulting lattice constant of $5.3 \AA$ implies a sizeable tensile strain of $+18 \%$ compared to buckled $\mathrm{Bi}(111)$ bilayers. This causes a fully planar configuration of the honeycomb rings. The synthesis (described in Methods) starts from a hydrogen-etched $\mathrm{SiC}$ wafer on which a monolayer of $\mathrm{Bi}$ is epitaxially deposited, giving rise to sharp electron diffraction peaks (Fig. E2). A scanning tunneling microscopy (STM) overview (Fig. 1b) shows that the whole surface is smoothly covered with flakes of typical diameter $\sim 25 \mathrm{~nm}$, separated by phase-slip domain boundaries (related to the Bi-induced surface reconstruction), and including occasional defects. Alternatively, the flakes are also terminated by SiC substrate steps, see Fig. 1c. Inspection of the layer on a smaller scale reveals the characteristic honeycomb pattern of bismuthene (Fig. 1d). Detailed scrutiny of the honeycomb structure is provided by the close-up STM in Fig. 1e. For both occupied and empty states, respectively, the images show a honeycomb pattern throughout. 
To establish that the electronic structure of the synthesized material corresponds to bismuthene, we performed angle-resolved photoelectron spectroscopy (ARPES) and compared it with density-functional theory (DFT), see Fig. 2. The electron bands in Fig. 2a are obtained using a hybrid exchange-correlation functional, including SOC (see Methods). At the K-point, the direct energy gap amounts to $1.06 \mathrm{eV}$ ( six orders of magnitude larger than in graphene with $\sim 1 \mu \mathrm{eV} 2$ ). The conduction band minimum at $\Gamma$ leads to an indirect gap of $0.67 \mathrm{eV}$ in DFT. The k-resolved dispersion from ARPES in Fig. 2b exhibits the characteristic maximum at the K-point and a band splitting there, in close agreement with the DFT overlay. From the ARPES close-up in Fig. 2c of the valence band maximum at $\mathrm{K}$, we derive a large band splitting of $\sim 0.43 \mathrm{eV}$. The constant energy maps in Fig. $2 \mathrm{~d}$ reflect the energy degeneracy of the $\mathrm{K}$ - and $\mathrm{K}$ '-points and give further proof of the high degree of long-range order in the Bi honeycomb lattice. The excellent agreement of ARPES and DFT results, thus, confirms the realization of a single bismuthene layer on SiC.

Next, we disentangle the key mechanisms that determine the energetics near the Fermi level $E_{\mathrm{F}}$ by reducing (downfolding) the full DFT band structure to the relevant low-energy model (see Supplementary Information for details). Inclusion of the substrate bonding proves indispensable in this procedure. We decompose the band structure into $\sigma$-bond contributions formed by $\mathrm{Bi} 6 s^{-}, p_{x^{-}}$ and $p_{y}$-orbitals, and $\pi$-bond contributions formed by $p_{z}$-orbitals. As a consequence of hybridization with the substrate via $\pi$-bonds, the orbital content at low energies is predominantly of $\sigma$-type (Fig. 3a). This manifests "orbital filtering", where the $p_{x^{-}}$and $p_{y^{-}}$orbitals play the pivotal role close to $E_{\mathrm{F}}$, while the $p_{z}$-orbital is projected out of this energy region.

Focussing on the low-energy bands around the Fermi level, we find that without relativistic 
effects the mere orbital hybridization in the $\sigma$-bonding sector (with eight bands due to two-fold orbital, spin, and sublattice degrees of freedom) yields a Dirac-like band crossing at the K point, see Fig. 3b. SOC comes into play via two leading contributions: First, the local (on-site) SOC term $\lambda_{\text {SOC }} L_{z} \sigma_{z}$ generates large matrix elements between $p_{x^{-}}$and $p_{y^{-}}$-orbitals, and directly determines the magnitude of the energy gap at the K-point (Fig. 3c), which is of order $\sim 2 \lambda_{\text {SoC }}$. Second, the $\pi$-bonding sector hybridizes with the substrate, which breaks inversion symmetry. It features a Rashba term which, in leading order of perturbation theory, couples into the $\sigma$-bonding sector, yielding a composite effective Hamiltonian:

$$
H_{\mathrm{eff}}^{\sigma \sigma}=H_{0}^{\sigma \sigma}+\lambda_{\mathrm{SOC}} H_{\mathrm{SOC}}^{\sigma \sigma}+\lambda_{\mathrm{R}} H_{\mathrm{R}}^{\sigma \sigma}
$$

where $H_{0}^{\sigma \sigma}, H_{\mathrm{SOC}}^{\sigma \sigma}$, and $H_{\mathrm{R}}^{\sigma \sigma}$ are specified in the Supplementary Information.

We derive the prefactors for $\mathrm{Bi} / \mathrm{SiC}$ as $\lambda_{\mathrm{SOC}} \sim 0.435 \mathrm{eV}$ and $\lambda_{\mathrm{R}} \sim 0.032 \mathrm{eV}$. Although the Rashba term $\lambda_{\mathrm{R}}$ is much smaller than $\lambda_{\mathrm{SOC}}$, it induces the large valence band splitting into states of opposite spin character, while leaving the conduction band spin-degenerate at K (Fig. 3d). The six valence band maxima are energy-degenerate, yet carry inverted spin character at the K- versus K'points, respectively ${ }^{23}$. Their large momentum separation and the huge Rashba splitting prevents spin scattering in the bulk ${ }^{24}$, as discussed for 2D semiconductors.

The mechanism at work here is very different from the Kane-Mele mechanism ${ }^{1}$ for graphene. Its gap emerges due to SOC between next-nearest neighbours and at the level of second-order perturbation theory - and is therefore minute. In other group-IV monolayers, such as silicene, the SOC remains of next-nearest neighbour type $\mathrm{e}^{25}$, and the relevant orbital is still $p_{z}$. In contrast, bismuthene represents the first realized honeycomb material in which the low-energy physics is 
driven by the huge on-site SOC of $p_{x}$ and $p_{y}$-orbitals. This is also distinguished from $\mathrm{HgTe} / \mathrm{CdTe}$ quantum wells, where the small band gap is governed by a complex band situation and opens only upon $\operatorname{strain}^{3 / 4}$.

Another fundamental ingredient making the wide-gap QSH phase possible is the tensile strain regime $(+18 \%)$ of bismuthene on $\mathrm{SiC}$. As argued for functionalized stanene ${ }^{10}$, the topological gap is lost when external strain shifts another band through the gap. This phase transition to a trivial system is promoted by compressive strain, while tensile strain stabilizes the QSH phase. Not surprisingly, compressed germanene $e^{\sqrt{13}}$ and stanene $e^{14}$ are reported as metals, which underpins the importance of the substrate.

A landmark feature of QSH systems are the helical edge channels, connecting valence and conduction states by two bands of opposite spin (Fig. E5), irrespective of the edge architecture (zigzag or armchair). Within the bulk gap, they exhibit an approximately constant density of states (DOS). In a ribbon simulation, the states rapidly decay towards the bulk within one unit cell, i.e. $\sim 5 \AA$ (Fig. E5).

Using a tunneling tip, the local DOS (LDOS) is inspected at the atomic scale. Bismuthene edges exist at $\mathrm{SiC}$ substrate steps. Differential tunneling conductivity ( $\mathrm{d} I / \mathrm{d} V$ ) curves, reflecting the LDOS, have been recorded along a path towards an uphill step in Fig. 4a. Far from the edge, the spectrum evidences the large bulk gap of $\sim 0.8 \mathrm{eV}$. Closer to the boundary, a state emerges filling the entire gap - as in the ribbon model. Its signal increases continuously towards the edge, while retaining its shape. A suppression of $\mathrm{d} I / \mathrm{d} V$ around zero bias might be a tip-related anomaly, or hint at Luttinger behaviour of the $1 \mathrm{D}$ edge states ${ }^{26}$, which requires further study. The edge DOS 
in Fig. 4b shows equivalent behaviour for upper and lower terrace. The $\mathrm{d} I / \mathrm{d} V$ signal (integrated over the gap) in Fig. 4c shows a decay length of $(4 \pm 1) \AA$, matching well with DFT for simple ribbon edges (Fig. E6).

These results bear important differences to a single Bi layer: free-standing it is hypothesized as QSH phase ${ }^{177}$ with metallic edge states ${ }^{16}$, which is presumed stable for up to several $\mathrm{Bi}$ layers ${ }^{15 / 27}$. Edge states for a single $\mathrm{Bi}$ layer on $\mathrm{Bi}_{2} \mathrm{Te}_{3}$ of $\sim 2 \mathrm{~nm}$ extent have been detected ${ }^{28}$ within a small gap of $\sim 70 \mathrm{meV}$, yet with $E_{\mathrm{F}}$ in the substrate valence band. $\mathrm{In}_{\mathrm{Bi}} \mathrm{Bi}_{14} \mathrm{Rh}_{3} \mathrm{I}_{9}$ edge states are also found, but $E_{\mathrm{F}}$ is pinned in the bulk conduction band ${ }^{29}$. In contrast, $E_{\mathrm{F}}$ resides within both the bismuthene gap $(\sim 0.8 \mathrm{eV})$ as well as the $\mathrm{SiC}$ gap $(3.2 \mathrm{eV})$, so that conduction is solely governed by the edge states.

While the topological character of the edge states has experimentally yet to be established, e.g., by a direct measurement of the QSH effect with its universal quantized conductance, the remarkable coherence of experimental evidence and theoretical prediction already makes a strong case for the QSH scenario in $\mathrm{Bi} / \mathrm{SiC}$. For $\mathrm{HgTe}^{4 / 5}$ the gap is $\sim 30 \mathrm{meV}$, and in $\mathrm{InAs} / \mathrm{GaSb} / \mathrm{AlSb}$ quantum wells ${ }^{6}$ merely $\sim 4 \mathrm{meV}$, which necessitates experiments below $300 \mathrm{mK}$. The key problem are "charge puddles", where defects push $E_{\mathrm{F}}$ into the bulk bands, overriding the $1 \mathrm{D}$ channel ${ }^{7 / 8}$. By comparison, the large-gap bismuthene suggests to be operational at RT. The domain size of $\sim 25 \mathrm{~nm}$ is expected to be increased by common technology. The accessible active layer enables many groundbreaking experiments, such as the response of the edge state to magnetic impurities, or quantum anomalous Hall setups ${ }^{\sqrt{30}}$ by chemical functionalization. 
Online Content Methods, along with any additional Extended Data display items and Supplementary Information, are available in the online version of the paper; references unique to these sections appear only in the online paper. 
1. Kane, C. L. \& Mele, E. J. Quantum spin Hall effect in graphene. Phys. Rev. Lett. 95, 226801 (2005).

2. Kane, C. L. \& Mele, E. J. $Z_{2}$ topological order and the quantum spin Hall effect. Phys. Rev. Lett. 95, 146802 (2005).

3. Bernevig, B. A., Hughes, T. L. \& Zhang, S.-C. Quantum spin Hall effect and topological phase transition in HgTe quantum wells. Science 314, 1757-1761 (2006).

4. König, M. et al. Quantum spin Hall insulator state in HgTe quantum wells. Science 318, 766-770 (2007).

5. Roth, A. et al. Nonlocal transport in the quantum spin Hall state. Science 325, 294-297 (2009).

6. Knez, I., Du, R.-R. \& Sullivan, G. Evidence for helical edge modes in inverted InAs/GaSb quantum wells. Phys. Rev. Lett. 107, 136603 (2011).

7. König, M. et al. Spatially resolved study of backscattering in the quantum spin Hall state. Phys. Rev. X 3, 021003 (2013).

8. Väyrynen, J. I., Goldstein, M. \& Glazman, L. I. Helical edge resistance introduced by charge puddles. Phys. Rev. Lett. 110, 216402 (2013).

9. Liu, C.-C., Feng, W. \& Yao, Y. Quantum spin Hall effect in Silicene and two-dimensional germanium. Phys. Rev. Lett. 107, 076802 (2011). 
10. Xu, Y. et al. Large-gap quantum spin Hall insulators in tin films. Phys. Rev. Lett. 111, 136804 (2013).

11. Lalmi, B. et al. Epitaxial growth of a silicene sheet. Applied Physics Letters 97 (2010).

12. Vogt, P. et al. Silicene: Compelling experimental evidence for graphenelike two-dimensional Silicon. Phys. Rev. Lett. 108, 155501 (2012).

13. Zhang, L. et al. Structural and electronic properties of germanene on $\mathrm{MoS}_{2}$. Phys. Rev. Lett. 116, 256804 (2016).

14. Zhu, F.-F. et al. Epitaxial growth of two-dimensional stanene. Nature Mat. 14, 1020 (2015).

15. Liu, Z. et al. Stable nontrivial $Z_{2}$ topology in ultrathin Bi (111) films: A first-principles study. Phys. Rev. Lett. 107, 136805 (2011).

16. Wada, M., Murakami, S., Freimuth, F. \& Bihlmayer, G. Localized edge states in twodimensional topological insulators: Ultrathin Bi films. Phys. Rev. B 83, 121310 (2011).

17. Murakami, S. Quantum spin Hall effect and enhanced magnetic response by spin-orbit coupling. Phys. Rev. Lett. 97, 236805 (2006).

18. Zhou, M. et al. Epitaxial growth of large-gap quantum spin Hall insulator on semiconductor surface. Proc. Natl. Acad. Sci. 111, 14378 (2014).

19. Hsu, C.-H. et al. The nontrivial electronic structure of $\mathrm{Bi} / \mathrm{Sb}$ honeycombs on $\mathrm{SiC}(0001)$. New Journal of Physics 17, 025005 (2015). 
20. Kuzumaki, T. et al. Re-investigation of the Bi-induced $\operatorname{Si}(111)-(\sqrt{3} \times \sqrt{3})$ surfaces by lowenergy electron diffraction. Surf. Sci. 604, 1044 (2010).

21. Miwa, R. H., Schmidt, T. M. \& Srivastava, G. P. Bi covered Si(111) surface revisited. Journal of Physics: Condensed Matter 15, 2441 (2003).

22. Yao, Y., Ye, F., Qi, X.-L., Zhang, S.-C. \& Fang, Z. Spin-orbit gap of graphene: First-principles calculations. Phys. Rev. B 75, 041401 (2007).

23. Xiao, D., Yao, W. \& Niu, Q. Valley-contrasting physics in graphene: Magnetic moment and topological transport. Phys. Rev. Lett. 99, 236809 (2007).

24. Xiao, D., Liu, G.-B., Feng, W., Xu, X. \& Yao, W. Coupled spin and valley physics in monolayers of $\mathrm{MoS}_{2}$ and other group-vi dichalcogenides. Phys. Rev. Lett. 108, 196802 (2012).

25. Liu, C.-C. et al. Low-energy effective hamiltonian for giant-gap quantum spin Hall insulators in honeycomb x-hydride/halide (x=N-Bi) monolayers. Phys. Rev. B 90, 085431 (2014).

26. Voit, J. One-dimensional fermi liquids. Reports on Progress in Physics 58, 977 (1995).

27. Takayama, A., Sato, T., Souma, S., Oguchi, T. \& Takahashi, T. One-dimensional edge states with giant spin splitting in a bismuth thin film. Phys. Rev. Lett. 114, 066402 (2015).

28. Yang, F. et al. Spatial and energy distribution of topological edge states in Single Bi (111) bilayer. Phys. Rev. Lett. 109, 016801 (2012).

29. Pauly, C. et al. Subnanometre-wide electron channels protected by topology. Nature Phys. 11, $338(2015)$ 
30. Niu, C. et al. Functionalized bismuth films: Giant gap quantum spin Hall and valley-polarized quantum anomalous Hall states. Phys. Rev. B 91, 041303 (2015).

Acknowledgements This work was supported by the Deutsche Forschungsgemeinschaft (DFG) under Grant SCHA1510/5, the SPP 1666 Priority Program ”Topological Insulators“, the DFG Collaborative Research Center SFB 1170 "ToCoTronics" in Würzburg, as well as by the European Research Council (ERC) through ERC-StG-Thomale-336012 "Topolectrics“. G. L. acknowledges the computing time granted at the Leibniz Supercomputing Centre (LRZ) in Munich. F.R. acknowledges many helpful discussions with M. R. Scholz and J. Aulbach.

Contributions J. S. conceived the experiment, S. G., F. R., M. B., and J. S. developed the material growth process. S. G. took the intial STM data. F. R. recorded all STM and STS data shown. L. D. conducted the photoelectron spectroscopy. DFT was done by G. L., and theoretical analysis jointly by G. L., R. T. and W. H. All authors contributed to interpreting the data. The manuscript was written by J. S., R.T., W. H., and R. C. with suggestions from all other authors.

Competing Interests The authors declare no competing financial interests.

Correspondence Correspondence and requests for materials should be addressed to J. S. (joerg.schaefer@ physik.uniwuerzburg.de). 
a

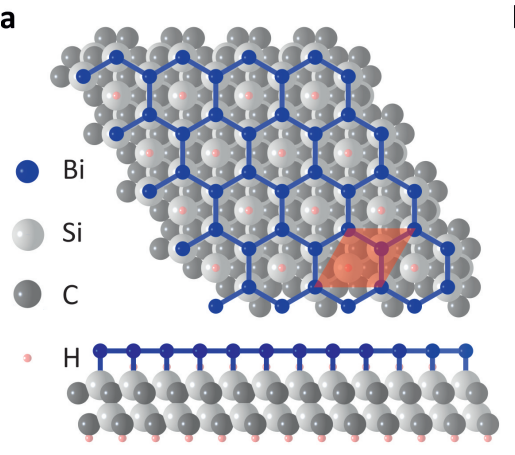

d

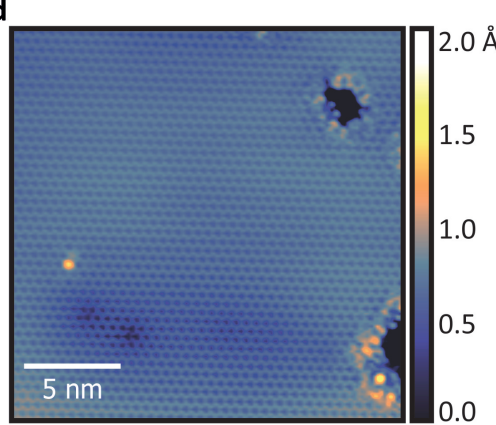

b

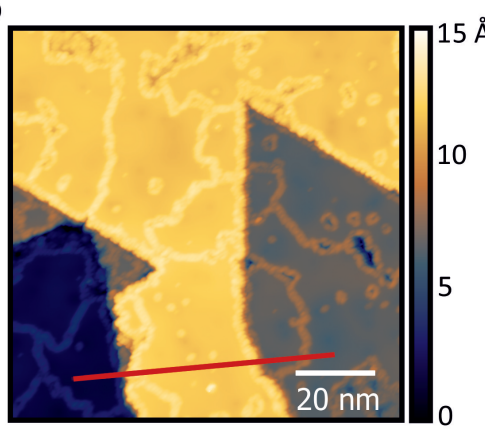

$15 \AA^{c}$

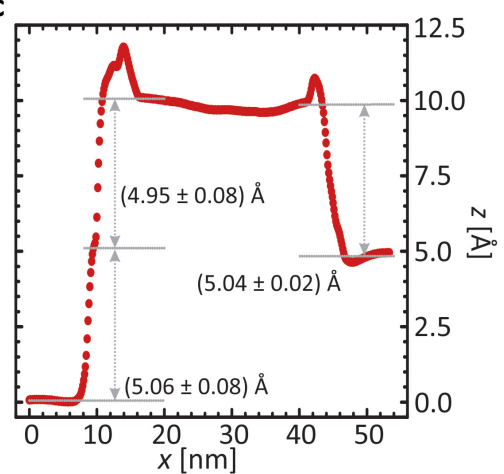

e
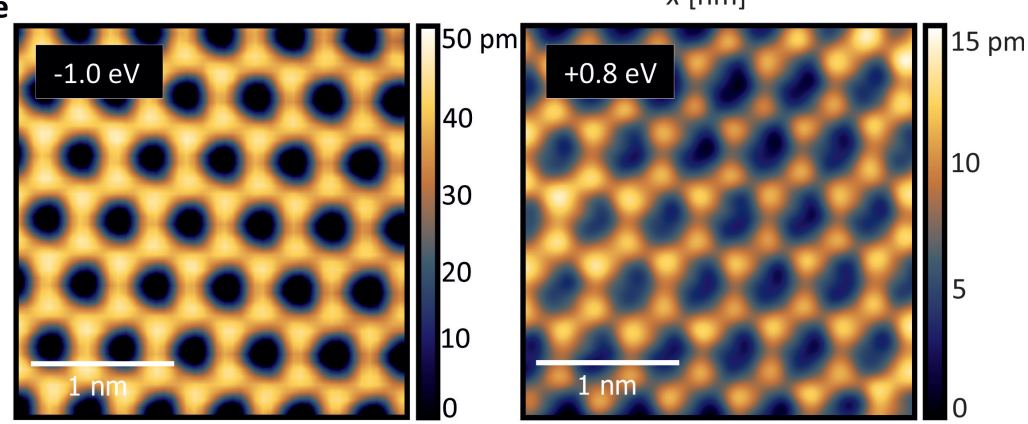

Figure 1 Structure Model and STM of Bi on SiC

Figure 1 Bismuthene on $\mathrm{SiC}(0001)$ structural model. a, Bismuthene layer placed on the threefold-symmetric $\mathrm{SiC}(0001)$ substrate in $(\sqrt{3} \times \sqrt{3}) \mathrm{R} 30^{\circ}$ commensurate registry.

b, Topographic STM overview map showing that bismuthene fully covers the substrate. The flakes are of $\sim 25 \mathrm{~nm}$ extent, limited by domain boundaries. c, Substrate step height profile, taken in $\mathbf{b}$. The step heights correspond to SiC steps. $\mathbf{d}$, The honeycomb pattern is seen on smaller scanframes. e, Close-up STM images for empty and occupied states. They confirm the formation of Bi honeycombs. 

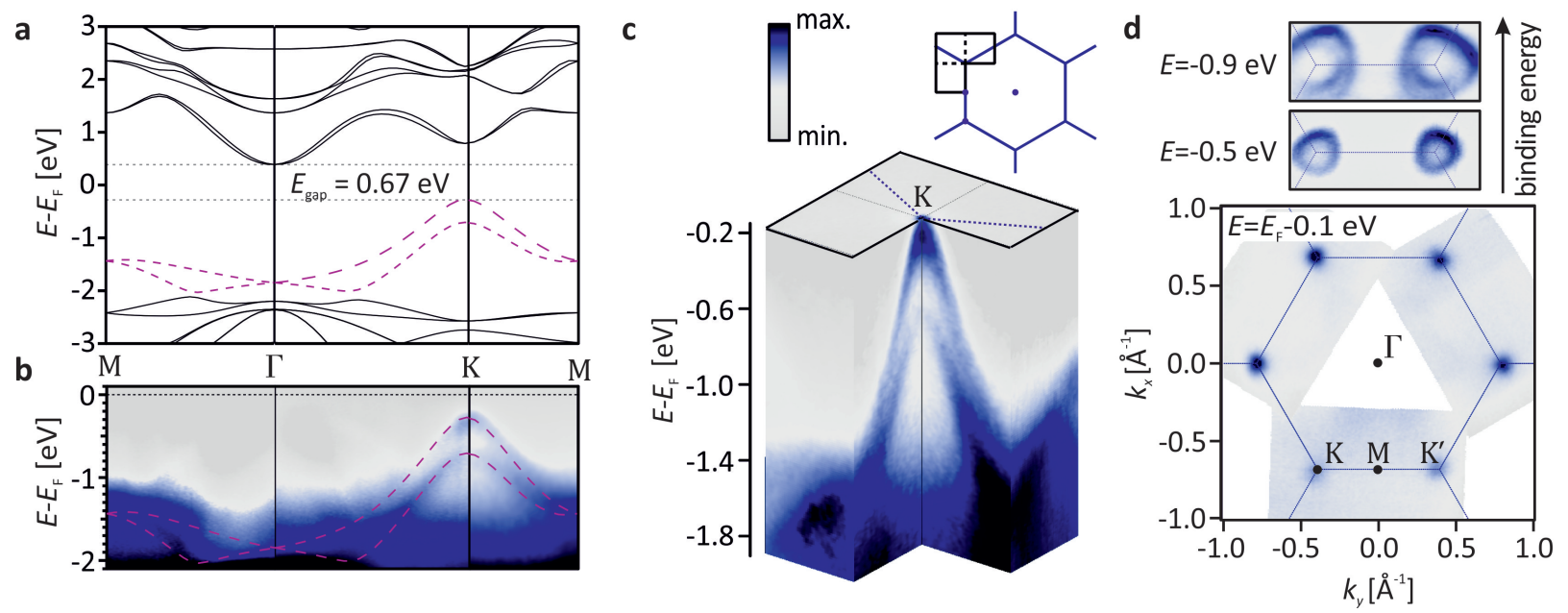

Figure 2 Theoretical band structure and ARPES measurements. a, DFT band structure calculation (using a HSE exchange functional) including SOC, showing the wide band gap and a significant band splitting in the valence band (dashed line). b, ARPES band dispersion through the Brillouin zone. The band maximum at $\mathrm{K}$ and the valence band splitting are in close agreement with the theoretical prediction (overlay). The zero of energy $\left(E_{F}\right)$ is aligned to the Fermi level of the spectrometer. $\mathbf{c}$, Valence band maximum at the K-point with large SOC-induced splitting in a wide momentum range. d, Constant energy surfaces from ARPES at various binding energies. The cut at low binding energies is taken at the topmost intensity corresponding to the valence band maximum. The sixfold degeneracy of the K- and K'-points of the hexagonal lattice is thereby confirmed. 

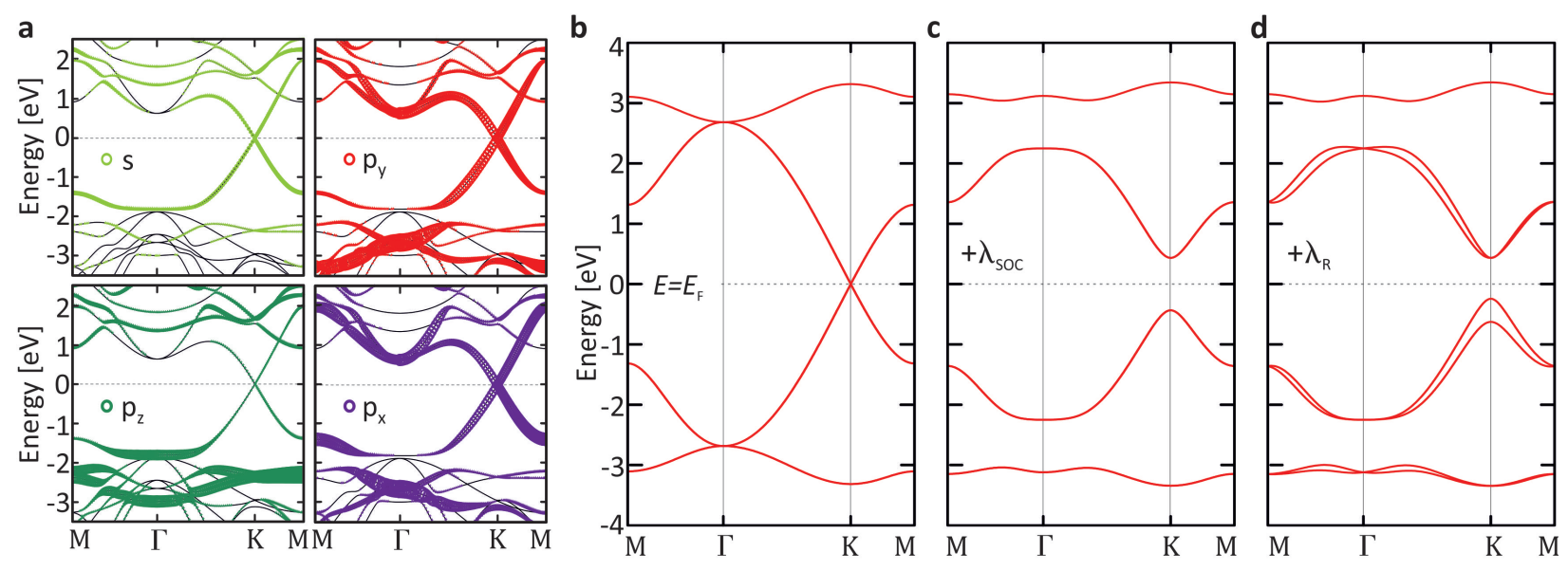

Figure 3 Orbital character and electronic structure of low-energy effective model of $\mathrm{Bi} \sigma$-bands

Figure 3 Orbital decomposition (w/o SOC) and the electronic structure of the low-energy effective model of $\mathbf{B i} \sigma$-bands. a, The contribution of $\mathrm{Bi} s$ and $p$-orbitals (without SOC) to the electronic structure (w/o SOC) of bismuthene. In each panel, the circle size is proportional to the relative weight of the orbital. $\ln \mathrm{Bi} / S i C, p_{x}$ - and $p_{y}$-orbitals prevail around $E_{F}$.

b. Electronic structure of the low energy effective model without SOC. c, Inclusion of the strong atomic SOC opens a huge gap at the K-point. d, Further including the Rashba term lifts the degeneracy of the topmost valence band, and induces a large splitting with opposite spin character there. 

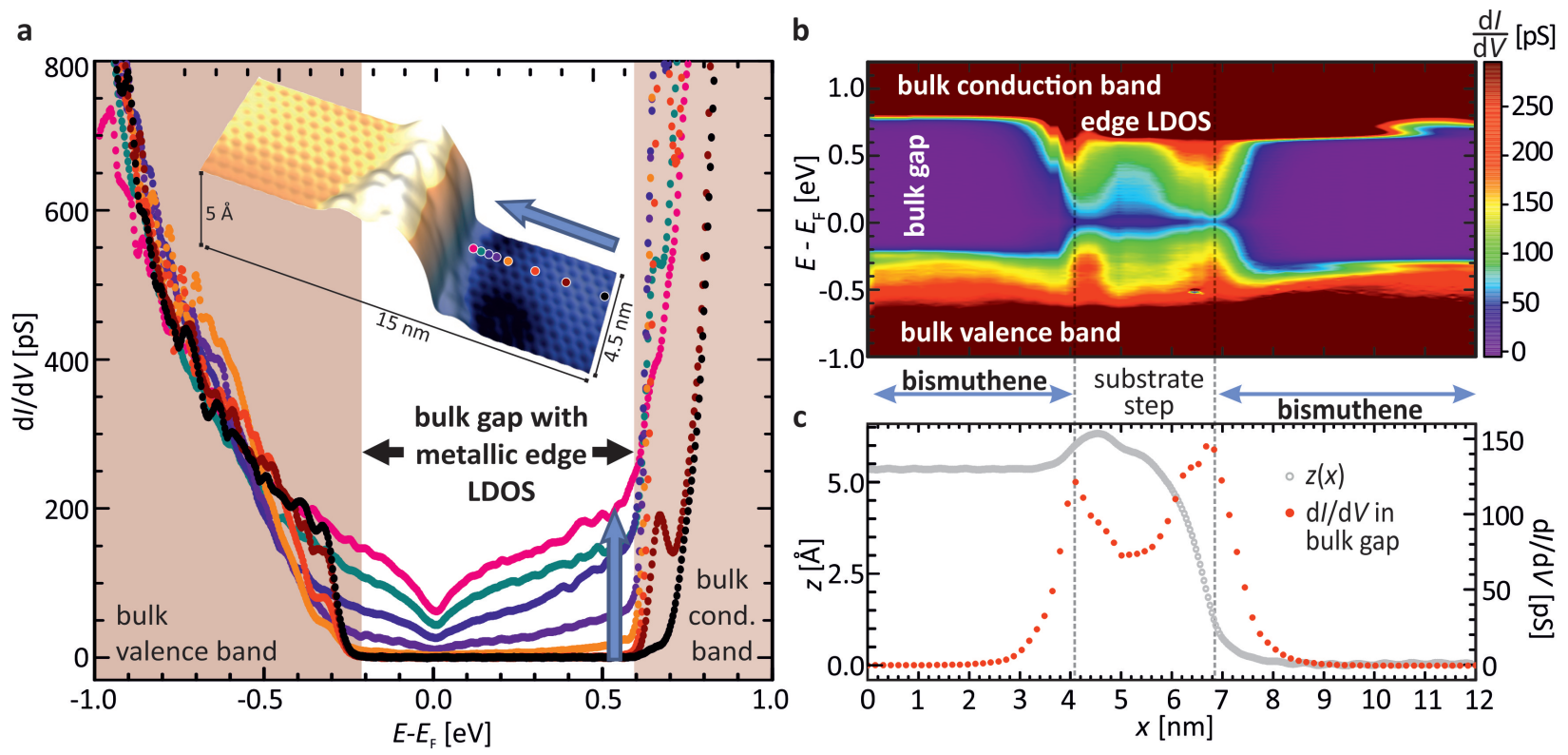

Figure 4 Spectroscopy of the Edge State

Figure 4 Tunneling spectroscopy of edge states at substrate steps. a, Differential conductivity $\mathrm{d} I / \mathrm{d} V$ (reflecting the LDOS) at different distances to the edge. A large gap of $\sim 0.8 \mathrm{eV}$ is observed in bulk bismuthene (black curve). Upon approaching the edge, additional signal of increasing strength emerges that fills the entire gap. Inset: STM measurement locations (color-coded dots relate to spectrum color) at uphill substrate step causing the boundary. b, Spatially resolved $\mathrm{d} I / \mathrm{d} V$ data across the same step. The $\mathrm{d} I / \mathrm{d} V$ signal of the in-gap states peaks at both film edges (grey dashed lines mark $\mathrm{d} I / \mathrm{d} V$ maxima). c, Topographic $z(x)$ line profile of the step, and $\mathrm{d} I / \mathrm{d} V$ signal of bismuthene (integrated over the gap from +0.1 to $+0.4 \mathrm{eV}$ ), showing an exponential decrease away from the step edge, on either side. 